\title{
Magnetic Hysteresis at the Domain Scale of a Multi-Scale Material Model for Magneto-Elastic Behavior
}

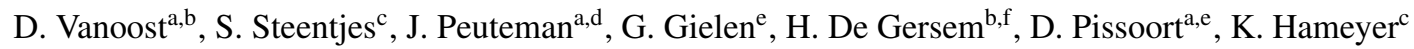 \\ ${ }^{a}$ KU Leuven Technology Campus Ostend, ReMI Research Group, Oostende B-8400, Belgium \\ ${ }^{b}$ KU Leuven Kulak, Wave Propagation and Signal Processing Research Group, Kortrijk B-8500, Belgium \\ ${ }^{c}$ Institute of Electrical Machines, RWTH Aachen University, Aachen D-52062, Germany \\ ${ }^{d}$ KU Leuven, Department of Electrical Engineering, Electrical Energy and Computer Architecture, Heverlee B-3001, Belgium \\ ${ }^{e}$ KU Leuven, Department of Electrical Engineering, Microelectronics and Sensors, Heverlee B-3001, Belgium \\ ${ }^{f}$ TU Darmstadt, Institut für Theorie Elektromagnetischer Felder, Darmstadt D-64289, Germany
}

\begin{abstract}
This paper proposes a multi-scale energy-based material model for poly-crystalline materials. Describing the behaviour of polycrystalline materials at three spatial scales of dominating physical mechanisms allows accounting for the heterogeneity and multiaxiality of the material behaviour. The three spatial scales are the poly-crystalline, grain and domain scale. Together with appropriate scale transitions rules and models for local magnetic behaviour at each scale, the model is able to describe the magneto-elastic behaviour (magnetostriction and hysteresis) at the macroscale, although the data input is merely based on a set of physical constants. Introducing a new energy density function that describes the demagnetisation field, the anhysteretic multi-scale energy-based material model is extended to the hysteretic case. The hysteresis behaviour is included at the domain scale according to the micromagnetic domain theory while preserving a valid description for the magneto-elastic coupling. The model is verified using existing measurement data for different mechanical stress levels.
\end{abstract}

Keywords:

Magnetostatics, nonlinear magnetics, magneto-mechanical couplings, hysteresis loops, multi-scale modelling, micro-magnetics.

\section{Introduction}

Modeling of ferromagnetic material behaviour is commonly done using microscopic approaches based on the LandauLifschitz-Gilbert equation or using macroscopic models with constitutive laws identified from macroscopic measurements. However, these attempts are either unfeasible for modelling macroscopic structures or do not consider the heterogeneity and multi-axiality of the material behaviour.

Contemporary electro-technical design requires highly accurate models for ferromagnetic materials. They should be capable of representing hysteresis and magnetostriction effects properly. Moreover, they should consider the multi-axiality and heterogeneity of the material behaviour. Finally, it should be possible to extract macroscopic effective properties from the constituent properties, local anisotropy and crystallographic texture. A multi-scale material model, connecting various spatial material scales upholding strong physical connections while remaining applicable as a design tool, is required.

In 1865 Villari found the correlation between a magnetisation change and the tensile stress of iron-based materials [34, 43], known as the Villari effect [12]. Buckley showed in 1925 [8] that a mechanical stress has a non-negligible impact on the magnetic material properties. It affects both the size as well as the shape of the hysteresis loop, i.e., the extrinsic properties coercivity and remanence $[7,8,13,16,28,29,30,38,35]$.

The internal mechanical stress can originate from: (i) the manufacturing process [5]; (ii) a heating or a cooling step [39]; (iii) the operation of the electrical device (e.g. centrifugal forces due to the high-rotational speed) [6]; or (iv) the magnetostriction in a poly-crystalline material [14].

In order to account for the magneto-elastic coupling two approaches can be distinguished based on their underlying physical assumptions.

1. Phenomenological macroscopic models predict the magnetic behaviour at the poly-crystalline scale introducing the mechanical stress as a parameter in a classical macroscopic hysteresis model, e.g., the Jiles-Atherton-Sablik model [37] or the Preisach model [1]. Other macroscopic models use thermodynamic arguments [24] as an additional macroscopic energy term including the magnetoelastic effect. Their disadvantages are: (i) the small working range, (ii) only representing isotropic materials, (iii) requiring tedious parameter identifications and (vi) mostly excluding multi-axial mechanical stresses. This means that they are acceptable for standard magnetic materials in traditional situations and are comparably simple with fast computations, but not appropriate as an investigation tool to optimize materials in specific designs. Moreover, they neglect the subtle coupling of magnetostriction and the magneto-elastic effect by excluding the knowledge of the internal domain structure and the crystallographic magnetic texture. 
(1) Poly-crystalline scale

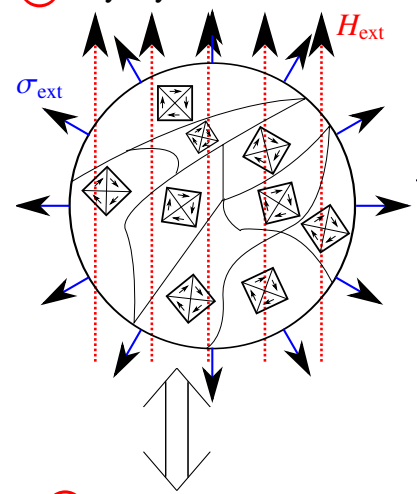

(2) Grain scale

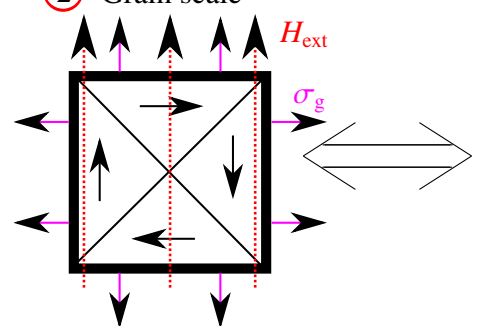

Texture data simulation

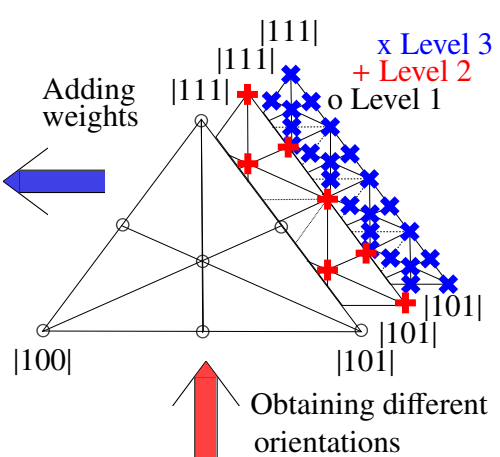

(3) Domain scale

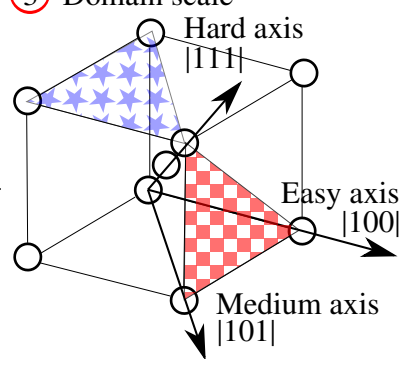

Figure 1: Various spatial material scales

2. Micro-magnetic models predict the magnetic behaviour at the domain scale and/or the grain scale. The magnetic domain behaviour, investigated by the energy density functions, represents the potential energy of the investigated crystal $[18,32,42]$. The minimum of the energy density functions defines current magnetic state being affected by a internal mechanical stress and a magnetic field. Among others, Armstrong [2] and DeSimone [17], use uni-axial and cubic crystalline symmetries in magneto-elastic simulations. These simulations are done on the domain structure limiting the approach in size to reduce the computational cost.

The aforementioned strategies are either of limited prediction range or difficult to manage to predict the result of the intricate magneto-elastic coupling of a poly-crystalline material for arbitrary mechanic stresses (vectorial, compressive, tensile).

In contrast, multi-scale methods are able to resolve the complexity of the magneto-elastic coupling by enriching the macroscopic material description by observations at various spatial scales. Describing the behavior of poly-crystalline materials at different spatial scales allows distinguishing between dominating physical mechanisms and accounting for the heterogeneity and multiaxiality of the material behavior. Together with appropriate scale transitions rules and models for the local magnetic behavior at each scale, the model is able to describe the magneto-elastic behavior (magnetostriction and hysteresis) at the macroscale although the data input is merely based on a set of physical constants.

This is possible using energy-based material models such as the Armstrong model [2] or the multi-scale model [15], when accomplished by a static hysteresis model. The Armstrong model uses an incremental hysteresis model for a single-crystal material [4], i.e., hysteresis is included for a fixed crystal direction by calculating the losses due to the crystal defects. In contrast the originally anhysteretic multi-scale approach presented in[15] is extended to the hysteretic case using the Hauser energetic model for ferromagnetic hysteresis for the description of poly-crystalline materials [16, 21]. Hysteresis is obtained by adding an irreversible magnetic field to the anhysteretic field component obtained by the anhysteretic multi-scale model [15]. This approach requires four additional free parameters identified by a tedious parameter identification. The multi-scale approach in [15] is based on the results of the multi-scale approach of: (i) poly-crystalline materials for structural mechanic simulations [23] and (ii) ferroelectric poly-crystallines [25], which are included in the non-linear behaviour of a heterogeneous material.

In this paper, however, the hysteresis effect is implemented at the domain scale for a poly-crystalline isotropic material. In contrast to [20], where artificial thresholds prevent early switching of magnetic domains, the Boltzmann distribution is extended using the grain scale magnetisation of the previous time step for correcting the statistical distribution of magnetic domains in the new energy density function. This approach requires less parameters compared to the Preisach [10], JilesAtherton [44] and Hauser [21] models, while including the effect of the mechanical stress on the hysteresis and does not demand a tedious parameter identification work.

The paper is organised as follows. Sec. 2 introduced the definition of the three different spatial material scales used to account for the magneto-elastic coupling affected by the different grain orientations in a poly-crystalline material as discussed in Sec. 3. Sec. 4 discusses the multi-scale model posed by Eshelby inclusion problem and the scale transitions rules mapping between the domain scale, the grain scale and the polycrystalline scale. Sec. 4.2 describes the three standard energy density functions of micromagnetism, i.e., Zeemann, magnetocrystalline anisotropy and magneto-elastic energy. In addition, the proposed energy density function for inclusion of magnetic hysteresis at the domain scale is presented. The model is validated in Sec. 5 using measurements and physical theories both available in literature. The paper ends with some conclusions.

\section{Various Spatial Material Scales}

The multi-scale material model iterates between three different spatial material scales to represent the magnetic behavior of the poly-crystalline material. The scales are the poly-crystalline scale, the grain scale and the domain scale, see Fig. 1.

1. The poly-crystalline scale $(0.1 \mathrm{~mm}-50 \mathrm{~mm})$ is a representative volume element [15], i.e., it represents the macroscopic material properties of the considered material. The poly-crystalline scale contains different, randomly oriented grains. Each grain contributes to the macroscopic behaviour.

2. One grain $(1 \mu \mathrm{m}-10 \mathrm{~mm})$ consists of multiple crystals, which are almost perfectly aligned with each other. The grain scale considers homogeneous elastic properties and 
a uniform strain [15]. The grain magnetisation is determined by the magnetic domains in the grain.

3. A magnetic domain $(0.1 \mu \mathrm{m}-1 \mu \mathrm{m}$ forms a substructure of the grain structure. Inside a magnetic domain both the mechanic as well as magnetic quantities are homogeneous, forming the smallest scale of the multi-scale scheme. Depending on the crystal structure, i.e., the crystal system, (i) body centred cubic (bcc) crystal, (ii) the face centred cubic (fcc) crystal, (iii) the hexagonal crystal, easy and hard magnetization directions are formed defining the direction of spontaneous domain magnetization in the demagnetized state $[7,9,10,12]$.

\section{Different Grain Orientations - Crystallographic Texture}

The relation between the magnetic and mechanic properties at the three different spatial scales is defined by a combination of localisation and homogenisation operations. Therewith it is possible to calculate the magnetisation and magnetostriction of a material point at the poly-crystalline scale accounting for the intricate magneto-elastic coupling ruled by characteristics described at different spatial scales.

The external magnetic field $H_{\text {ext }}$ and mechanical stress $\sigma_{\text {ext }}$ act on the poly-crystalline scale and are described in the same reference frame, called the poly-crystalline reference frame. This reference frame is the main reference frame in the material model. The other reference frames used at the grain scale are determined by the different grain orientations. In contrast to [15], the magnetic field acting at the grain scale is not transformed in a localisation scheme to include the effect of the demagnetisation field, but transformed to the reference of the grain scale. Instead, the effect of the demagnetisation field is included using a new energy density function at the domain scale, see Section 4.2 .

The mechanical stress $\sigma_{\mathrm{g}}$ at the grain scale consists of the mechanical stress $\sigma_{\text {ext }}$ applied externally and a component that accounts for the interaction of the different grains with each other obtained using a localization scheme. This localisation scheme is based on solving Eshelby's inclusion problem, as described in $[15,19,31]$. The Eshelby inclusion problem is solved for a poly-crystalline material consisting of multiple grains considered as different inclusions [15, 19, 31]. This approach relies on the macroscopic crystallographic magnetic texture of the material, implying the different orientations of the grains/inclusions are known. Instead of measuring the orientation distribution function (ODF) of one single specific sample [26] and assuming that this is representative for the whole material an ODF is constructed for the whole material.

As described in [41], a cubic crystal consists of 24 indistinguishable standard triangles. A standard triangle is a plane cornered by the directions $|100|,|101|,|111|$. As shown in Fig. 1, scale 3, the red checker board triangle can be transformed into another standard triangle (e.g., the blue star triangle in Fig. 1, scale 3) using an appropriate rigid rotation of the crystal. This rotation of the crystal does not influence the atomic arrangements, but enables to reduce the number of grain orientations by distributing the grain orientations in one standard triangle. Indeed, when the magnetic field is aligned with a direction in the blue star triangle in Fig. 1, scale 3, the effect on the crystal equals the effect when the magnetic field aligns with the similar direction in the red checker board triangle in Fig. 1, scale 3 , apart from rotation [27]. This decreases significantly the required number of grain orientations that have to be considered and speeds up the model while maintaining the same accuracy.

In order to simulate a magnetically isotropic material, a uniform distribution of the grains is needed. This uniform distribution is obtained by mapping the different grain orientations in an equilateral triangle, see Fig. 1, red vertical arrow. Depending on the required accuracy, different levels can be used. The equilateral triangle with corners $|100|,|101|,|111|$, has the practical property to generate a uniform distribution. Every point in this map represents a grain. The externally applied magnetic field is aligned with the specific grain direction shown in the map. This specific grain orientation and the uniform distribution are obtained using the vertices, middle faces and centroids of the equilateral triangle. In order to obtain a more accurate model, a higher number of grain orientations needs to be considered. This could be done iteratively to obtain a certain, predefined accuracy, i.e., not limited to four. Depending on the level $L$ of accuracy, $N_{\text {\#triangles }}$ identical equilateral triangles are used, leading to $N_{\# \text { orientations }}$ different grain orientations.

$$
\begin{aligned}
N_{\text {\#triangles }} & =4^{L-1} \\
N_{\text {\#orientations }} & =\left(3+\left(2^{L}-2\right)\left(1-0^{L-1}\right)\right) !+4^{L-1}
\end{aligned}
$$

The use of accuracy level one, two or three, requires to divide an equilateral triangle into one, four or sixteen identical equilateral triangles leading to seven, nineteen or sixty-one different grain orientations which are uniformly distributed by construction. For level one accuracy, the used grain orientations are the directions where the magnetic field aligns with the vertices $(|100|,|101|,|111|)$, middle faces $(|201|=|100|+$ $|101|,|211|=|100|+|111|,|212|=|101|+|111|)$ and centroids $(|321|=|100|+|101|+|111|)$. Middle faces in accuracy level $L-1$ become vertices in accuracy level $L$. When material with magneto-crystalline anisotropy is studied, different weights can be assigned to the different grains at the poly-crystalline scale (see Fig. 1, blue horizontal arrow).

\section{Multi-scale Material Model}

The multi-scale material model (Fig. 2), uses the external magnetic field $H_{\text {ext }}$ (point 1 in Fig. 2), the external mechanical stress $\sigma_{\text {ext }}$ (point 2 in Fig. 2), some material parameters (point 3 in Fig. 2) and a prescribed accuracy as inputs. The material parameters are composed of five physical constants characteristic for the unit cell of the crystal system: (i) the saturation magnetisation $M_{\mathrm{s}}$, (ii) the magneto-crystalline anisotropy constants $K_{1}$ and $K_{2}$, (iii) the saturation magnetostrictions $\lambda_{100}$ and $\lambda_{111}$ of the crystal systems unit cell along the $|100|$ and $|111|$ directions, (iv) the elasticity coefficients $C_{11}, C_{12}$ and $C_{44}$. 


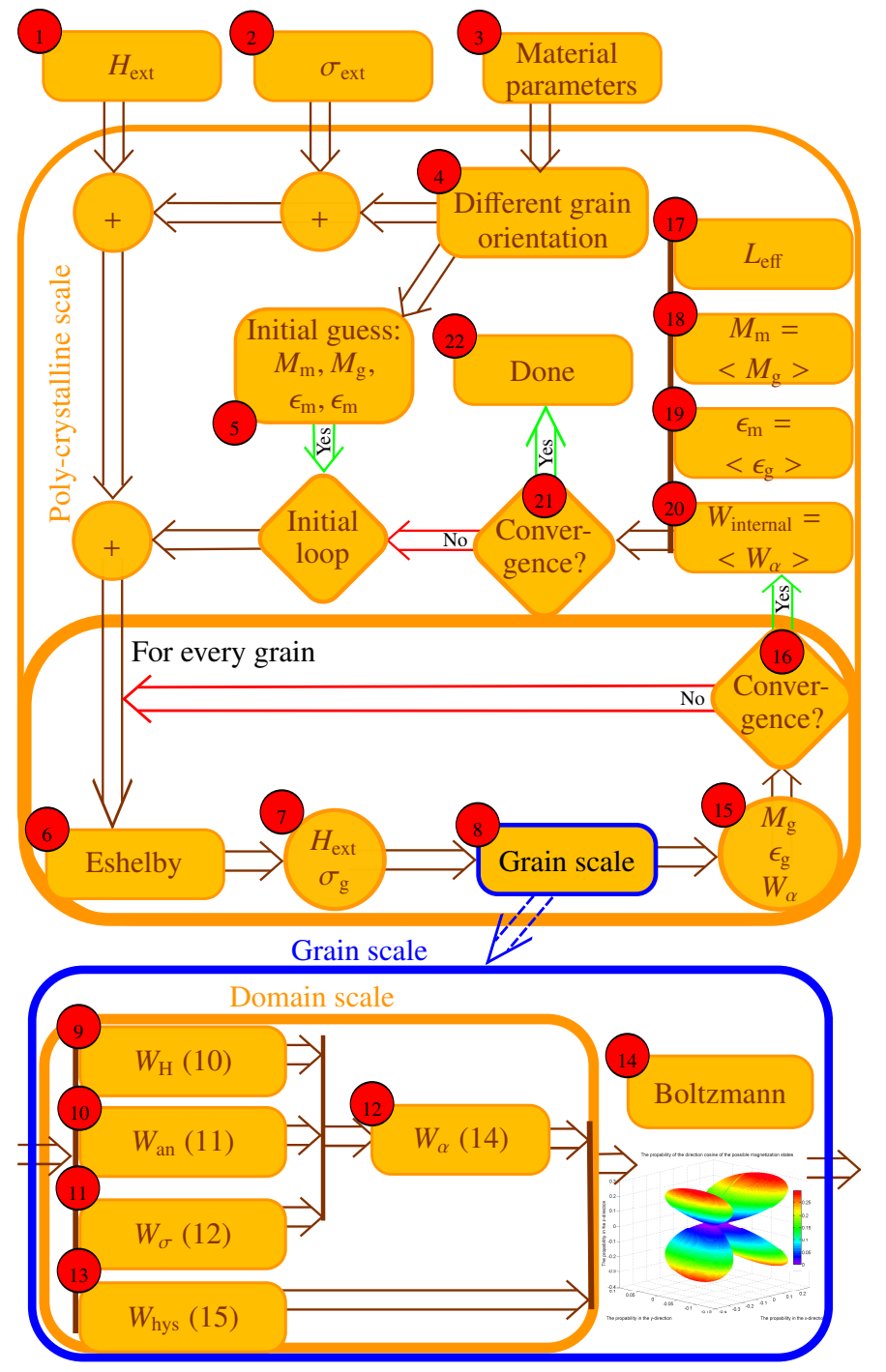

Figure 2: Flowchart of the material model detailing the different localisation and homogenization operations using the external magnetic field $H_{\text {ext }}$ and mechanical stress $\sigma_{\text {ext }}$ as inputs. Point 8 is worked out in detail in points 9 to 14 . The output of the model is the poly-crystalline magnetisation $M_{\mathrm{m}}$ and magnetostriction $\epsilon_{\mathrm{m}}$.

Based on the material parameters, the different grain orientations are modelled (point 4 in Fig. 2), as described in Section 2 leading to the initial guesses of: (i) the mean poly-crystalline scale magnetisation $M_{\mathrm{m}}$, (ii) all grain scale magnetisations $M_{\mathrm{g}}$, (iii) the mean poly-crystalline scale magnetostriction $\epsilon_{\mathrm{m}}$ and (iv) all grain scale magnetostrictions $\epsilon_{\mathrm{g}}$ (point 5 in Fig. 2).

\subsection{Localization: Eshelby's inclusion problem}

The effective elastic and magnetic properties of the polycrystalline material depend on the properties, interactions and orientations of the individual grains. In order to account for the intricate coupling at the grain scale a transformation of the applied mechanical stress and magnetic field from the polycrystalline scale to the grain scale is required. The grain scale mechanical stress $\sigma_{\mathrm{g}}$ originates in parts from (i) the external mechanical stress $\sigma_{\text {ext }}$, (ii) the magnetostrictive strains due to

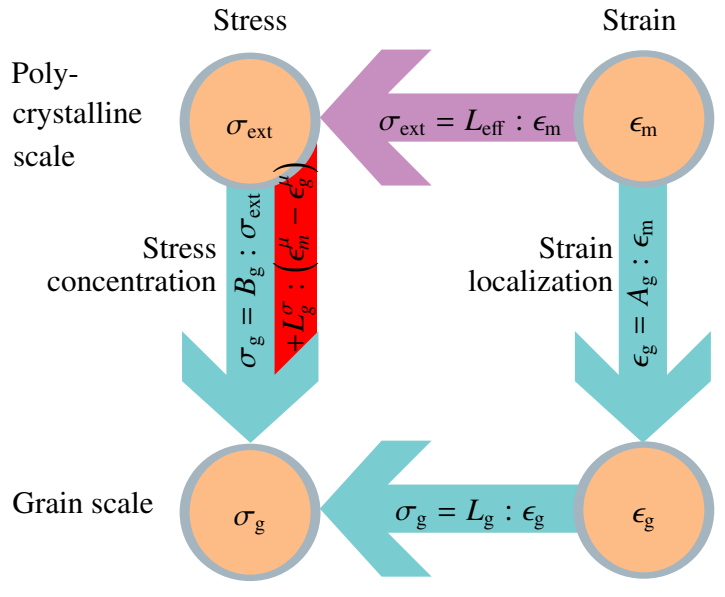

Figure 3: Details of the localization and concentration operation, performed in point 6 of Fig. 2, used to solve the Eshelby inclusion problem.

the applied magnetic field and (iii) the collisions between the grains.

In order to include the interaction of the different grains with each other, a self-consistent scheme (Fig. 2) is used $[11,15,16,33]$. As described in $[15,16,19,31]$, passing from grain to poly-crystalline quantities represents a typical problem of homogenisation that requires a self-consistent polycrystalline scheme. This is a homogenisation procedure based on the work of Hill [31], Kröner [31] and Eshelby [19]. It assumes that the elastic properties are known on the grain scale (point 8 in Fig. 2) and starts from equations (3) and (4), where $B_{\mathrm{g}}$ and $A_{\mathrm{g}}$ are, respectively, the stress-concentration and the strain-localization tensor (point 6 of Fig. 2). Starting from the situation at the previous time step an iteration between the grain and poly-crystalline scale is conducted until a consistent strain distribution is obtained [33]. The result is the required grain scale mechanical stress $\sigma_{\mathrm{g}}$ (point 7 in Fig. 2).

Fig. 3 shows in more detail the localization and concentration scheme used in point 6 of Fig. 2. The grain scale mechanical stress $\sigma_{\mathrm{g}}$ is composed of the external mechanical stress $\sigma_{\text {ext }}$ (transformed to the crystal reference frame) and the residual mechanical stress. The residual mechanical stress originates from the eigenstrain, i.e., the free strain (magnetostrictive strain). This generated residual mechanical stress requires that the condition $\sigma_{\text {ext }}=\left\langle\sigma_{\mathrm{g}}\right\rangle=L_{\text {eff }}: \epsilon_{\mathrm{m}}$ is fulfilled, where $L_{\text {eff }}$ is the effective elasticity coefficient tensor of the poly-crystalline material and $\epsilon_{\mathrm{m}}$ is the poly-crystalline scale strain (point 17 in Fig. 2).

$$
\begin{aligned}
\sigma_{\mathrm{g}} & =B_{\mathrm{g}}: \sigma_{\mathrm{ext}} \\
\epsilon_{\mathrm{g}} & =A_{\mathrm{g}}: \epsilon_{\mathrm{m}}
\end{aligned}
$$

Notice that (4) is only the first part of the equation used in Fig. 3. The second part of this equation extends (4) to the magneto-elastic coupling. The stress-concentration $B_{\mathrm{g}}$ and the strain-localization $A_{\mathrm{g}}$ tensors, which are fourth-order tensors that function as linear operators, are defined by [15]:

$$
B_{\mathrm{g}}=L_{\mathrm{g}}: A_{\mathrm{g}}: L_{\mathrm{eff}}^{-1}
$$




$$
\begin{aligned}
A_{\mathrm{g}} & =\left(L_{\mathrm{g}}+L_{*}\right)^{-1}:\left(L_{\mathrm{eff}}+L_{*}\right) \\
L_{*} & =L_{0}:\left(S_{\mathrm{E}}^{-1}-I\right)
\end{aligned}
$$

In order to calculate the stress-concentration $B_{\mathrm{g}}$ and the strainlocalization $A_{\mathrm{g}}$ tensors, Hill's constraint tensor $L_{*}(7)$ is needed. Hill's constraint tensor is determined by: (i) the Eshelby tensor $S_{\mathrm{E}}$, (ii) the fourth order unit tensor $I$ and (iii) the elasticity coefficient tensor $L_{0}$ of the homogeneous material. $L_{0}$ has the same properties as the poly-crystalline material. Eshelby [19] has shown that, assuming spherical inclusions, the Eshelby tensor $S_{\mathrm{E}}$ only depends on the elasticity coefficient. This means that $L_{0}$ is the only variable. The self-consistent poly-crystalline scheme [33] works by setting $L_{0}=L_{\text {eff }}$ and calculating $L_{\text {eff }}$ using (8) at the poly-crystalline scale (point 17 in Fig. 2).

$$
L_{\mathrm{eff}}=\left\langle L_{\mathrm{g}}:\left(L_{\mathrm{g}}+L_{*}\right)^{-1}:\left(L_{\mathrm{eff}}+L_{*}\right)\right\rangle
$$

Equation (8), which is central for the self-consistent scheme, is an implicit equation. To solve this implicit equation an iteration process is required.

The self-consistent poly-crystalline scheme can be extended to the magneto-elastic coupling (red part of the stress concentration in Fig. 3), by including the magnetic interaction in (3). The magnetic interaction is implemented, using the effect of the magnetostrictive strain on the grain scale $\epsilon_{\mathrm{g}}^{\mu}$ and the polycrystalline scale $\epsilon_{\mathrm{m}}^{\mu}$, forming an eigenstress, which amounts to replacing (3) by (9).

$$
\sigma_{\mathrm{g}}=B_{\mathrm{g}}^{\sigma}: \sigma_{\mathrm{ext}}+L_{\mathrm{g}}^{\sigma}:\left(\epsilon_{\mathrm{m}}^{\mu}-\epsilon_{\mathrm{g}}^{\mu}\right)
$$

Here, $L_{\mathrm{g}}^{\sigma}$ is the elastic incompatibility tensor. It is important to note that these tensors depend only on the elastic coefficients of the grain and the considered homogeneous medium. The elastic coefficients of the homogeneous medium are obtained from the elastic coefficients of the grain and the self-consistent poly-crystalline scheme. The elastic coefficients of the grain are considered to be constant (Section 2). Equation (9) incorporates the incompatibility due to the difference in the behaviour between an individual grain and the surrounding medium. Neither the actual poly-crystalline strain $\epsilon_{\mathrm{m}}$, nor the grain scale strain $\epsilon_{\mathrm{g}}$ are used. Instead the macroscopic magnetostrictive strain $\epsilon_{\mathrm{m}}^{\mu}$ and grain scale magnetostriction strain $\epsilon_{\mathrm{g}}^{\mu}$ are used. The magnetostrictive part of the strain is the part that represents the magnetic interaction, i.e., the strain due to the magnetic field which causes the residual mechanical stress. Only these magnetostrictive strains are used as the eigenstrain in the Eshelby inclusion problem. The macroscopic magnetostriction strain is obtained with a volume average over the polycrystal.

\subsection{Domain scale}

The grain scale mechanical stress $\sigma_{\mathrm{g}}$ and external magnetic field $H_{\text {ext }}$ used as inputs for the grain scale (point 8 in Fig. 2) directly serve as inputs for the domain scale calculation. Based on these, the corresponding energy densities are calculated. These are (i) the Zeeman energy density $W_{\mathrm{H}}$ (point 9 in Fig. 2), (ii) the magnetocrystalline anisotropy energy density $W_{\text {an }}$ (point 10 in Fig. 2) and (iii) the mechanical stress induced anisotropy energy density $W_{\sigma}$ (point 11 in Fig. 2) [10,
Chapter 7] as well as the hysteresis energy density function $W_{\text {hys }}$ (point 13 in Fig. 2). The assumptions of a uniform strain and a uniform magnetic field exclude the need of calculating the exchange energy [15].

\subsubsection{Anhysteretic approach for magneto-elastic coupling}

The anhysteretic description of the reversible magnetoelastic behaviour is based on the definition of the free energy of the material at the domain scale. In combination with the scale transition rules of Section 4.1 the behaviour of the poly-crystalline material is defined. The free energy $W_{\alpha}$ of a domain $\alpha$ is assumed to be uniform and is the sum of three contributions. The Zeeman energy density $W_{\mathrm{H}}(10)$ is the energy stored in the system due to the magnetic field $H=\left[H_{1}, H_{2}, H_{3}\right]$. The Zeeman energy density is minimized when the magnetisation $M_{\mathrm{S}}\left[\alpha_{\mathrm{M}_{1}}, \alpha_{\mathrm{M}_{2}}, \alpha_{\mathrm{M}_{3}}\right]$ has the same orientation as the magnetic field, i.e., it is the magneto-static energy tending to align the domain magnetization along the magnetic field. $\alpha_{\mathrm{M}}=\left[\alpha_{\mathrm{M}_{1}}, \alpha_{\mathrm{M}_{2}}, \alpha_{\mathrm{M}_{3}}\right]$ defines the direction cosines of the possible magnetisation states (point 9 of Fig. 2).

$$
W_{\mathrm{H}}=-\mu_{0} M_{\mathrm{s}}\left(\alpha_{\mathrm{M}_{1}} H_{1}+\alpha_{\mathrm{M}_{2}} H_{2}+\alpha_{\mathrm{M}_{3}} H_{3}\right)
$$

In order to account for the presence of directions of easy magnetization of the underlying crystal system [12, Chapter 7], the magneto-crystalline anisotropy energy density $W_{\text {an }}$ (11) is used (point 10 of Fig. 2). In case of a cubic crystallographic symmetry, the crystal anisotropic constants $K_{1}$ and $K_{2}$ are used.

$$
\begin{gathered}
W_{\text {an }}=K_{1}\left[\left(\alpha_{\mathrm{M}_{1}}\right)^{2}\left(\alpha_{\mathrm{M}_{2}}\right)^{2}+\left(\alpha_{\mathrm{M}_{2}}\right)^{2}\left(\alpha_{\mathrm{M}_{3}}\right)^{2}+\right. \\
\left.\left(\alpha_{\mathrm{M}_{3}}\right)^{2}\left(\alpha_{\mathrm{M}_{1}}\right)^{2}\right]+K_{2}\left[\left(\alpha_{\mathrm{M}_{1}}\right)^{2}\left(\alpha_{\mathrm{M}_{2}}\right)^{2}\left(\alpha_{\mathrm{M}_{3}}\right)^{2}\right]
\end{gathered}
$$

Interaction of mechanical stress and the crystal structure [12] affects the hard, medium and easy directions of magnetization. The stress induced anisotropy energy density $W_{\sigma}(12)$ (point 11 of Fig. 2) depends on the saturation magnetostrictions $\lambda_{100}$ and $\lambda_{111}$ in case of a cubic crystallographic symmetry. The saturation magnetostrictions are defined by the strain change due to the rotation of a fully random magnetisation state to a fully oriented magnetisation state in the |100| or |111| direction, respectively. Equation (12) is notated in the Mandel notation [22], which vectorizes the mechanical stress as $\sigma=\left[\sigma_{11}, \sigma_{22}, \sigma_{33}, \sigma_{23}, \sigma_{13}, \sigma_{12}\right]$.

$$
\begin{aligned}
W_{\sigma}= & -\frac{3}{2} \lambda_{100}\left[\sigma_{11}\left(\left(\alpha_{\mathrm{M}_{1}}\right)^{2}-\frac{1}{3}\right)+\sigma_{22}\left(\left(\alpha_{\mathrm{M}_{2}}\right)^{2}-\frac{1}{3}\right)\right. \\
& \left.+\sigma_{33}\left(\left(\alpha_{\mathrm{M}_{3}}\right)^{2}-\frac{1}{3}\right)\right]-3 \lambda_{111}\left[\sigma_{23}\left(\alpha_{\mathrm{M}_{2}} \alpha_{\mathrm{M}_{3}}\right)\right. \\
& \left.+\sigma_{13}\left(\alpha_{\mathrm{M}_{1}} \alpha_{\mathrm{M}_{3}}\right)+\sigma_{12}\left(\alpha_{\mathrm{M}_{1}} \alpha_{\mathrm{M}_{2}}\right)\right]
\end{aligned}
$$

Equation (12) assumes that the magnetostrictive strain is isochore [15]. This implies that the strain can be described with two instead of three parameters as shown in (13) in Mandel 


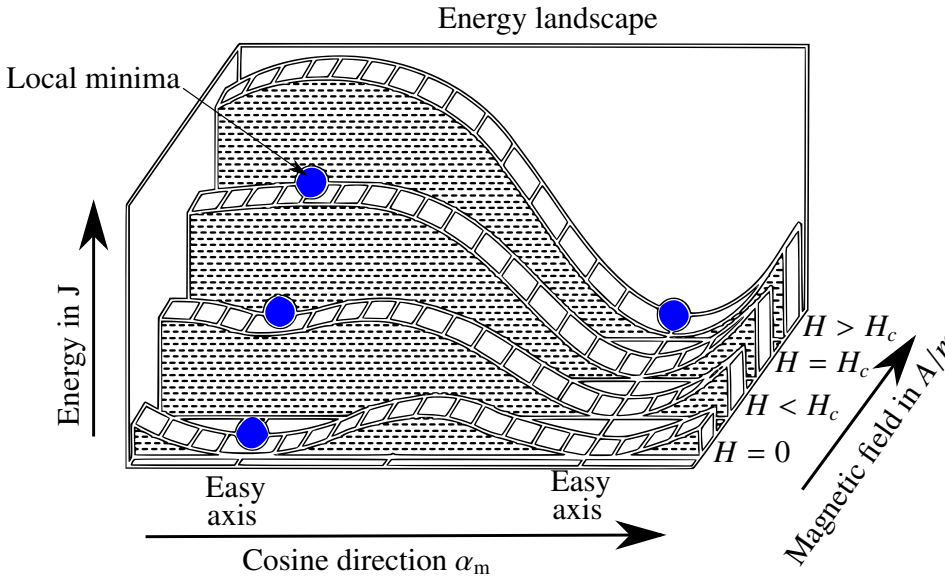

Figure 4: The energy landscape, showing the local minima explaining the remanent magnetisation and coercivity (figure adopted from [10])

notation [22]:

$$
\epsilon_{\mu}=\frac{3}{2}\left[\begin{array}{c}
\lambda_{100}\left(\gamma_{1}^{2}-1 / 3\right) \\
\lambda_{100}\left(\gamma_{2}^{2}-1 / 3\right) \\
\lambda_{100}\left(\gamma_{3}^{2}-1 / 3\right) \\
\lambda_{111} \sqrt{2} \gamma_{2} \gamma_{3} \\
\lambda_{111} \sqrt{2} \gamma_{1} \gamma_{3} \\
\lambda_{111} \sqrt{2} \gamma_{1} \gamma_{2}
\end{array}\right] .
$$

The sum of these three energy densities (14) depends on the direction cosines of the possible magnetisation states $\alpha_{\mathrm{M}}=\left[\alpha_{\mathrm{M}_{1}}, \alpha_{\mathrm{M}_{2}}, \alpha_{\mathrm{M}_{3}}\right]$. When the crystal structure is perfect, the magnetisation moments will align with the cosine direction $\gamma=\left[\gamma_{1}, \gamma_{2}, \gamma_{3}\right]$, which is the direction with the lowest total internal energy density $\left(W_{\alpha}(\gamma)=\min \left(W_{\alpha}\right)\right)$.

$$
W_{\alpha}=W_{\mathrm{H}}+W_{\mathrm{an}}+W_{\sigma} .
$$

\subsubsection{Hysteresis effects}

The described multi-scale model is restricted to the reversible part of the magneto-elastic behaviour so far. The complex, intricate coupling of elastic and magnetic effects is captured but irreversible effects are not included. In the following we propose to introduced the hysteresis effect at the domain scale. The irreversible behaviour is incorporated at the domain scale by adding an additional energy density to the free energy that accounts for the magnetic state of the previous time step. Depending on the applied magnetic field, the applied mechanical stress and the previous distribution state of the magnetic domains in the grain, the new state is obtained. When hysteresis occurs, the difference in magnetisation is mainly due to another distribution pattern. Indeed, the magnetisation equals zero for a grain which consists out of six equal domains aligned with the easy axis (in case the $|100|$ is an easy axis) and also when two equal opposite domains are much larger than the other four equal domains. The opposing force that wants to keep the magnetisation and distribution of the magnetic domain constant is described as a demagnetisation field that ensures that the magnetic domains want to be aligned with their nearest easy dir- ection of magnetization and could be interpreted as a mechanical analogy by inertia. Critical to this approach is the influence of the crystal structure on the shape of the hysteresis loop $[9,10]$. This resembles to the early work of Stoner and Wohlfarth [10, 40] that determines the shape of the hysteresis loop according to the angle between the magnetisation and the easy axis of the Stoner-Wohlfarth particle (uni-axial crystals). Although the Stoner-Wohlfarth model is quite out of context for cubic crystals, because a Stoner-Wohlfarth particle only has one easy axis, it demonstrates the importance of the easy axis for the hysteresis process.

In a cubic crystal there are three or four easy axes available leading to a different magnetisation process. In this case the focus lays on the domain wall motion, i.e., $90^{\circ}$ wall motion in materials which have the $|100|$ as an easy axis and $71^{\circ}$ and $109^{\circ}$ wall motion in materials which have the $|111|$ as an easy axis [10], when low magnetic fields are used. For higher magnetic fields, when most domains are favourably oriented, a reversible rotation occurs.

The mechanical stress affects the distribution of the magnetic domains by affecting the easy axis. Even when the stress induced anisotropy energy density is an order of magnitude lower than the magneto-crystalline anisotropy energy density, its effect is not negligible. This will lead to another distribution of the magnetic domains over the easy axes, because some easy axes are now more preferred than others due to the stress. The transition occurs due to wall motion, leading to the growth of better oriented magnetic domains at the expense of the other magnetic domains. As described in [9] this leads to the more rectangular shaped hysteresis loop, when the nearest easy axis to the magnetic field becomes more preferred and to the more diamond shaped hysteresis loop, when the nearest easy axis to the magnetic field becomes less preferred. When the applied magnetic field is sufficiently large to overcome an energy barrier between two stable states, all magnetic moments are forced to align with the magnetic field, as visualised in Fig. 4. When the magnetic field decreases again, the magnetic moments want to align with the easy axis nearest to the magnetic field. This is not always the original easy axis, giving rise to different magnetisation phenomena, which are responsible for the magnetic hysteresis effect.

In order to model the effect of a magnetic field on the domain evolution (orientation of magnetic moments), i.e., the $180^{\circ}$ wall motion or the non $180^{\circ}$ wall motion, when considering a single mechanical state, it is possible to use artificial thresholds preventing early switching of magnetic domains as proposed in [20]. However, when considering several mechanical states or a dynamic process this simple technique is not satisfactory. The artificial thresholds depend on the mechanical state in combination with the applied magnetic field because mechanical stress has a considerable effect on $90^{\circ}$ wall motion $(|100|$ as an easy axis) and $71^{\circ}$ and $109^{\circ}$ wall motion (|111| as an easy axis).

Instead of using artificial thresholds, in this paper, a hysteresis energy density function is introduced which accounts for the magnetisation distribution of the previous states interpreted as an analogy to inertia. The previous state determines the easy axis with a higher probability to be aligned with. The hyster- 
esis energy density function proposed in this paper is described as (point 13 in Fig. 2):

$$
W_{\mathrm{hys}}=-\mu_{0} \frac{M_{\mathrm{s}}}{\chi_{0}}\left(\alpha_{\mathrm{M}_{1}} M_{\mathrm{hys} 1}+\alpha_{\mathrm{M}_{2}} M_{\mathrm{hys} 2}+\alpha_{\mathrm{M}_{3}} M_{\mathrm{hys} 3}\right)
$$

It resembles to the Zeeman energy density and is based on the saturation magnetisation $M_{\mathrm{s}}$, the calculated initial grain scale magnetic susceptibility $\chi_{0}$ and the calculated grain scale magnetisation in the previous time step $M_{\text {hys }}$. Therewith, the offset with respect to the previous time step, which affects the behaviour of the magnetic moments, is taken into account. When the material is in a demagnetised state, the hysteresis energy density function equals zero for every possible cosine direction. This does not lead to a preferred direction due to the previous time step and the proposed model provides the same results as the anhysteretic model. However, when the material is in a saturated state, the hysteresis energy density function equals a distribution having a maximum and a minimum. The difference between the maximum and the minimum is the energy needed to enforce the flipping of the magnetic moments when the magnetic field is aligned with the easy axis, due to the $180^{\circ}$ wall motion. When the magnetic field is not aligned with an easy axis, the hysteresis energy density function ensures that the magnetic moments favour the easy direction nearest to the magnetic field.

The hysteresis energy density function is calculated at the domain scale, because not all easy directions of the different grains are aligned. However, there is also a different internal mechanical stress in every grain due to the residual mechanical stress. This affects the preferred easy directions in a grain. As will be described in detail in Section 4.3, the Boltzmann distribution applied to (15) accounts for crystal defects and pinning effects, which also play an important role in the hysteresis effect $[4,7,9,12,16]$. The proposed energy density function is used separately and increases the obtained probability for the anhysteretic part nearest to the grain scale magnetisation in the previous time step as described in Section 4.3 (point 13 of Fig. 2). In contrast to [15], $\chi_{0}$ is not measured as a single poly-crystalline constant but simulated for every individual grain using the first two time steps. $\chi_{0}$ is a scalar value instead of tensor that describes the linearised relation of the projection of the magnetisation on the magnetic field. Meaning $\chi_{0}$ depends on the angle between the magnetic field and the nearest easy axis. Because the easy axes have different orientation in every grain and since the poly-crystalline scale is seen as a single crystal aggregate, every grain should have its own $\chi_{0}$.

\subsection{Grain-scale}

Starting from the free energy $W_{\alpha}$ of a given domain, the distributions of the magnetic moments across the different orientations, i.e., their volume fractions, are introduced as internal variables according to an explicit Boltzmann-type relation. This results in the grain scale magnetisation $M_{\mathrm{g}}$ and magnetostriction $\epsilon_{\mathrm{g}}$ using the Boltzmann distributions $P_{\alpha}, P_{\text {hys }}$ and $P_{\mathrm{g}}$ (point 14 of Fig. 2). These distributions use an adjustable material parameter $A_{\mathrm{s}}$ which accounts for the domain walls, non-uniformity of mechanical stresses or magnetic fields and other defects in the crystal. As described in [15], this requires a
Boltzmann distribution, which is used as a statistical approach of a specific state by comparing the energy of one state to the energy of all other states instead of a simple minimization approach. Indeed, a minimization would only lead to the most preferred direction, but in reality not all magnetic domains will be aligned with this direction. Depending on the internal energy, determined by the three energy density functions introduced in Sec. 4.2.1, the distribution of the magnetic domains is determined based on the assumption that the previous distribution was an equal distribution across the easy axes, leading to the demagnetised state of the grain. This was shown by [15], leading to Eq. (16).

$$
\begin{aligned}
P_{\alpha} & =\frac{\exp \left(-A_{\mathrm{s}}\left(W_{\mathrm{H}}+W_{\mathrm{an}}+W_{\sigma}\right)\right)}{\int_{\alpha} \exp \left(-A_{\mathrm{s}}\left(W_{\mathrm{H}}+W_{\mathrm{an}}+W_{\sigma}\right)\right)} \\
P_{\text {hys }} & =\frac{\exp \left(-\beta A_{s} W_{\mathrm{hys}}\right)}{\int_{\alpha} \exp \left(-\beta A_{\mathrm{s}} W_{\mathrm{hys}}\right)} \\
P_{\mathrm{g}} & =\frac{P_{\alpha} P_{\text {hys }}}{\int_{\alpha} P_{\alpha} P_{\text {hys }}}
\end{aligned}
$$

The Boltzmann distribution $P_{\alpha}$ includes the parameter

$$
A_{\mathrm{s}}=\left(3 \chi_{\mathrm{m} 0}\right) /\left(\mu_{0} M_{\mathrm{s}}^{2}\right)
$$

as a measure of the regularity of the crystal. According to [15], the expression for $A_{\mathrm{s}}$ is obtained neglecting the rotation mechanism, which leads to a simplified model of the magnetic behaviour.

In this paper, the poly-crystalline scale magnetic susceptibility $\chi_{\mathrm{m} 0}$ is replaced by the simulated grain scale susceptibility $\chi_{0}$. The additional hysteresis energy density function (15), at the domain scale, includes the effect of magnetic hysteresis by increasing the probability of the anhysteretic part (16) closest to the grain scale magnetisation in the previous time step.

The inclusion of the hysteresis is done by using an additional Boltzmann distribution $P_{\text {hys }}$ in combination with a correction factor $\beta . \beta$ is used to fine tune the parameter $A_{\mathrm{s}}$ for the hysteretic case and the product $\beta A_{\mathrm{s}}$ acts as a potential distribution parameter [3]. This parameter determines the defocus of the local magnetisation along the average directions due to different crystal defects. When using only parameter $A_{\mathrm{s}}$, the results deviate substantially from measurements, indicating that some of the assumptions made in [15] to obtain the expression of $A_{s}$, are not completely valid for the hysteretic case, such as the rotation mechanism and the pinning effect [10, Chapter 7]. Hauser suggested in [21] that the irreversible work due to pinning (because domains are mainly covering pinning sites to reduce the internal energy) affects the probability density. Hauser resolved this using a parameter related to the pinning similar with the classical Maxwell and Boltzmann statistics. In our approach, we suggest a similar approach using the factor $\beta$ attributed anhysteretic model to the pinning effect which is not incorporated in parameter $A_{\mathrm{s}}$ [15]. The correction factor $\beta$ has an effect on the width of the hysteresis loop and is assumed to be a constant value of 0.875 in this paper.

Once the Boltzmann distributions are known for any direction of magnetization, the combination of both (18) allows to 
obtain the grain scale magnetization $M_{\mathrm{g}}$ and magnetostrictive strain $\epsilon_{\mathrm{g}}$ with a volume average over the single crystal (20) and (21). This step is obtained at point 15 in Fig. 2.

$$
\begin{gathered}
M_{\mathrm{g}}=\left[\begin{array}{c}
\int_{\alpha} P_{\mathrm{g}} \alpha_{\mathrm{M}_{1}} M_{\mathrm{s}} \\
\int_{\alpha} P_{\mathrm{g}} \alpha_{\mathrm{M}_{2}} M_{\mathrm{s}} \\
\int_{\alpha} P_{\mathrm{g}} \alpha_{\mathrm{M}_{3}} M_{\mathrm{s}}
\end{array}\right] \\
\epsilon_{\mathrm{g}}=\left[\begin{array}{c}
\int_{\alpha} P_{\mathrm{g}} \frac{3}{2} \lambda_{100}\left(\alpha_{\mathrm{M}_{1}}^{2}-\frac{1}{3}\right) \\
\int_{\alpha} P_{\mathrm{g}} \frac{3}{2} \lambda_{100}\left(\alpha_{\mathrm{M}_{2}}^{2}-\frac{1}{3}\right) \\
\int_{\alpha} P_{\mathrm{g}} \frac{3}{2} \lambda_{100}\left(\alpha_{\mathrm{M}_{3}}^{2}-\frac{1}{3}\right) \\
\int_{\alpha} P_{\mathrm{g}} \frac{3}{2} \lambda_{111}\left(\sqrt{2} \alpha_{\mathrm{M}_{2}} \alpha_{\mathrm{M}_{3}}\right) \\
\int_{\alpha} P_{\mathrm{g}} \frac{3}{2} \lambda_{111}\left(\sqrt{2} \alpha_{\mathrm{M}_{1}} \alpha_{\mathrm{M}_{3}}\right) \\
\int_{\alpha} P_{\mathrm{g}} \frac{3}{2} \lambda_{111}\left(\sqrt{2} \alpha_{\mathrm{M}_{1}} \alpha_{\mathrm{M}_{2}}\right)
\end{array}\right]
\end{gathered}
$$

\subsection{Poly-crystalline-scale}

When the magnetisation $M_{\mathrm{g}}$ and the magnetostriction $\epsilon_{\mathrm{g}}$ converged for all grains (point 16 in Fig. 2), a weighted averaging procedure is applied. The weights depend on the directions of the grains, which are assigned as indicated by the blue horizontal arrow in Fig. 1, using the constructed orientation distribution function described in Section 3. Here, the poly-crystalline scale magnetisation $M_{\mathrm{m}}$ (point 18 in Fig. 2), the magnetostriction $\epsilon_{\mathrm{m}}$ (point 19 in Fig. 2) and the internal energy $W_{\text {internal }}$ (point 20 in Fig. 2) are determined as well as the elasticity coefficient tensor $L_{\mathrm{eff}}$ of the equivalent homogeneous material using (8) (point 16 in Fig. 2). At this scale, a second convergence criterion (point 21 in Fig. 2), i.e., on the weighted average internal energy $W_{\text {internal, }}$, is verified which is needed to check the convergence of the self-consistent polycrystalline scheme.

\section{Modelling Results and Comparison to Measured Data}

The proposed multi-scale model with magnetic hysteresis at the domain scale is applied to model the behaviour of a commercially available non-oriented $\mathrm{FeSi} 3 \%$ electrical steel grade.

\subsection{Material parameters}

The material model is parametrised without tedious parameter identification work solely using data taken from the textbooks of Bozorth [7] and Cullity [12] as given in Table 1).

\begin{tabular}{|c|c|c|c|}
\hline$M_{\mathrm{s}}$ & $K_{1}$ & $K_{2}$ & $\lambda_{100}$ \\
\hline $1.61 \mathrm{MA} / \mathrm{m}$ & $38 \mathrm{~kJ} / \mathrm{m}^{3}$ & $0 \mathrm{~J} / \mathrm{m}^{3}$ & $23 \mu \mathrm{m} / \mathrm{m}$ \\
\hline$\lambda_{111}$ & $C_{11}$ & $C_{12}$ & $C_{44}$ \\
\hline$-4.5 \mu \mathrm{m} / \mathrm{m}$ & $202 \mathrm{GPa}$ & $122 \mathrm{GPa}$ & $229 \mathrm{GPa}$ \\
\hline
\end{tabular}

Table 1: Physical magnetic and mechanical constants [7, 12].

\subsection{Comparison with measurements from literature}

The results obtained using the material are validated against measurements found in literature [13, 16, 28, 29, 30, 38]. Fig. 5 shows simulated major loops for isotropic FeSi3\% with different applied mechanical stresses of 100, 50, 15, 0, -15, -50 and $-100 \mathrm{MPa}$. A positive mechanical stress represents a tensile stress, whereas a negative mechanical stress represents a compressive stress. The simulation is performed using the parameters of Table 1. It is apparent that the material model is able to simulate the effect of a tension or a pressure on the magnetisation as well as on the magnetostriction. Fig. 5a shows a qualitative agreement with Fig. 9 of [13] and Fig. 4 of [28] in case of a compressive stress. The effect of a pressure or a tension on the magnetisation originates from a different magnetisation mechanism as described in [9] and Section 4.2.2. According to [9] two "coincidence points" (points where all curves intersect) appear.

The results for the magnetostriction (Fig. 5b) show the expected butterfly loops as described in [4, 7, 12, 16]. Fig. 5b shows how an applied mechanical stress affects the shape of the hysteresis loop of the magnetostriction. The shape of the hysteresis loop of the magnetostriction is explained using the magnetic domains and how they are affected by the mechanical stress and the magnetic field. This will be clarified using two cases:

1. A high uni-directional tension on a positive magnetostrictive material, like $\mathrm{Fe}-\mathrm{Si} 3 \%$, leads to a high probability that the magnetic domains are already aligned with the mechanical stress direction. A magnetic field applied in the same direction, results in a flipping of magnetic moments, due to a $180^{\circ}$ domain wall. The magnetic moment will not rotate to another domain aligned with a different easy axis. When the magnetic moments prefer to stay on the same easy axis, implies that the moments do not contribute to the magnetostriction. Indeed, when a magnetic moment flips, the overall shape and the axes of the magnetic domain moments remain exactly the same.

2. A high uni-directional pressure on a positive magnetostrictive material, like $\mathrm{Fe}-\mathrm{Si} 3 \%$, leads to a higher probability that the magnetic domains are not aligned with the mechanical stress direction but in the perpendicular plane. A magnetic field applied in the same direction of the mechanical stress, leads to a $90^{\circ}$ rotation due to the wall motion, of the magnetic moments and no flip. The fact that the magnetic moments rotate instead of flipping, implies that they contribute to the magnetostriction. Indeed, when a magnetic moment rotates the overall shape of the magnetic domain moment is rotated. In case of a positive magnetostrictive material, this leads to an elongation in the magnetisation direction. The opposite, namely a shrink, occurs in the plane perpendicular to the magnetisation direction. This rotation only occurs when the magnetic field is high enough. The higher the mechanical pressure, the more domains that contribute to the magnetostriction, which leads to a higher magnetostriction. There is however a trade-off. Indeed, a higher pressure requires also a higher magnetic 
field in order to reach saturation, as shown in Fig. 5b. This means a higher pressure leads to a higher magnetostriction which is obtainable with a higher magnetic field.

Fig. 6 shows simulated minor loops using the proposed material model. The minor loops are obtained after performing a calibration process to obtain the origin of the magnetisation.

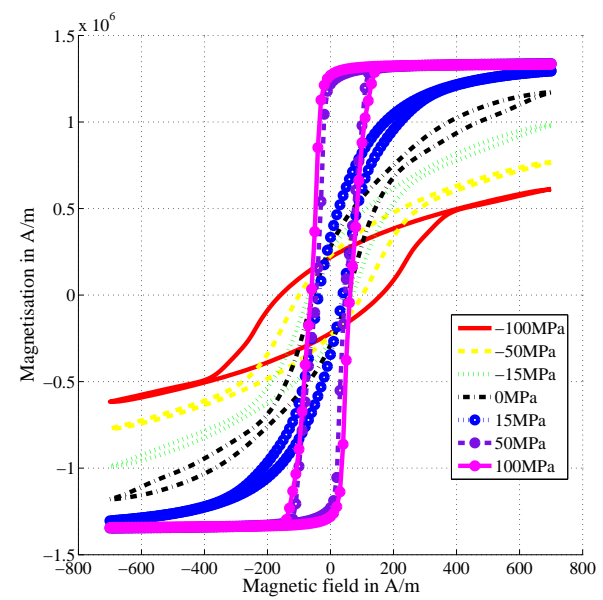

(a) Magnetisation

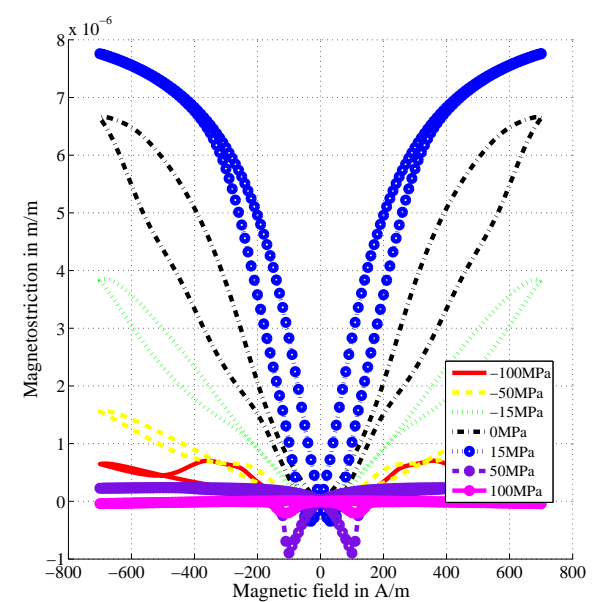

(b) Magnetostriction

Figure 5: Simulated major loops for isotropic FeSi3\% with different applied mechanical stresses of $100,50,15,0,-15,-50$ and $-100 \mathrm{MPa}$. A positive mechanical stress represents a tensile stress, whereas a negative mechanical stress represents a compressive stress. The simulation is performed using the parameters of Table 1.

These results show that the proposed material model simulates these magnetic material behaviour without the need to perform measurements and extract macroscopic models by tedious parameter identification. Fine-tuning of material parameters according to the specific characteristics of the material (crystallographic magnetic texture, internal mechanical stress state) is expected to provide an better agreement, but such an approach is not in the focus of the current paper.

The next step is to compare the model predictions based on the data from standard textbooks without any additional ad-

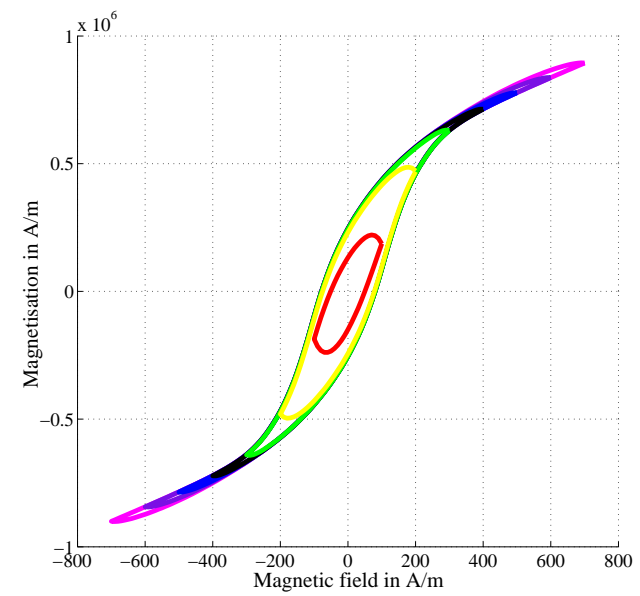

Figure 6: Simulated minor loops for a pressure of $50 \mathrm{MPa}$ with different amplitudes amplitude of 100, 200, 300, 400, 500600 and $700 \mathrm{~A} / \mathrm{m}$ of the applied magnetic field.

justment to measured data found in literature for a similar alloyed electrical steel grade. Fig. 7 shows a good qualitative agreement between the simulated results and the measurements available in literature [36] when a pressure is applied (compare Fig. 5 with Fig. 7). The proposed material model does not account for the non-monotonic effect a tensile stress. According to the classical theory of magneto-elasticity application of an uni-axial tensile stress to a positive magnetostrictive material results in a steeper, more rectangular hysteresis loop, i.e., magnetic saturation would be obtained faster. Instead experimental results show that the effect on the magnetic susceptibility is non-monotonic with stress intensity when going to higher tensile mechanical stress, e.g., from 50 to $100 \mathrm{MPa}$. This is in contradiction to the classical theory of the magneto-elastic effect, meaning that the same phenomenon is already present in the original anhysteresis model of Daniel et. al. [15] and is not included in the model so far. Recent proposals attribute that to a demagnetising effect due to the number of domains (not only volume fraction but also their distribution) or due the fact that some grains are already plastic at a certain threshold level of mechanical stress. Following the classical theory of the magneto-elastic effect, a further deformation of the hysteresis loops into a square loop, as proposed by Bozorth and Bulte in $[7,9]$ for materials with a positive magnetostriction, is obtained. The obtained square loop of the model is more similar to hysteresis loops found for a grain-oriented silicon steel as shown in Fig 8. The obtained square loop is explained by the fact that the grains are more oriented in the direction of the tension (which is the same direction as the magnetic field), resulting in similar properties as the grain-oriented silicon steel. Fig 8 visualizes measured major loops [34] for grain-oriented $\mathrm{Fe}-\mathrm{Si} 3 \%$. This feeds the expectation that the remaining differences are attributed to unknown crystallographic magnetic texture and unknown internal mechanical stresses, possibly introduced by the manufacturing process. 


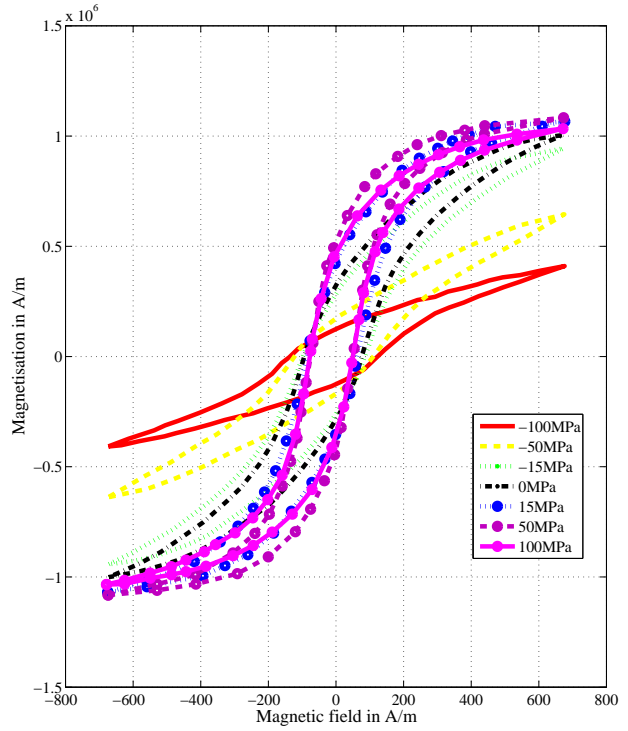

Figure 7: Measured major loops for non-oriented FeSi3\% with different applied mechanical stresses of $100,50,15,0,-15,-50$ and $-100 \mathrm{MPa}$ [36]. A positive mechanical stress represents a tensile stress, whereas a negative mechanical stress represents a compressive stress.

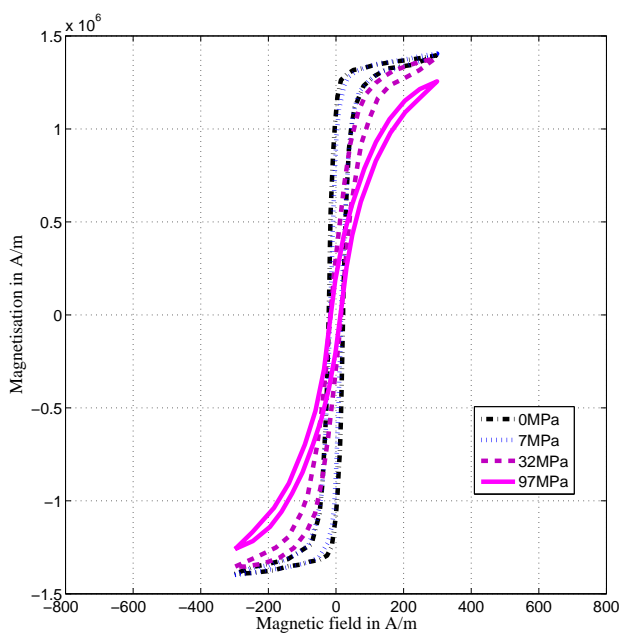

Figure 8: Measured major loops for grain-oriented FeSi3\% with different applied mechanical tensile stresses of 0, 7, 32 and $97 \mathrm{MPa}$ [34]. This graph is converted to SI units.

\subsection{Effect of the crystallographic magnetic texture}

Texture data describe the distribution of the grain orientations within the poly-crystalline sample. This distribution plays an important role in the magnetic behaviour of the material. Indeed, when all grains are perfectly aligned with each other, the sample behaves as a single crystal. The residual mechanical stress originating from the magnetostriction is reduced and, when properly used in a magnetic design, leads to small squareshaped hysteresis loops [9].

Fig. 9 and Fig. 10 show the effect of the crystallographic magnetic texture on the simulation results for a tension and a

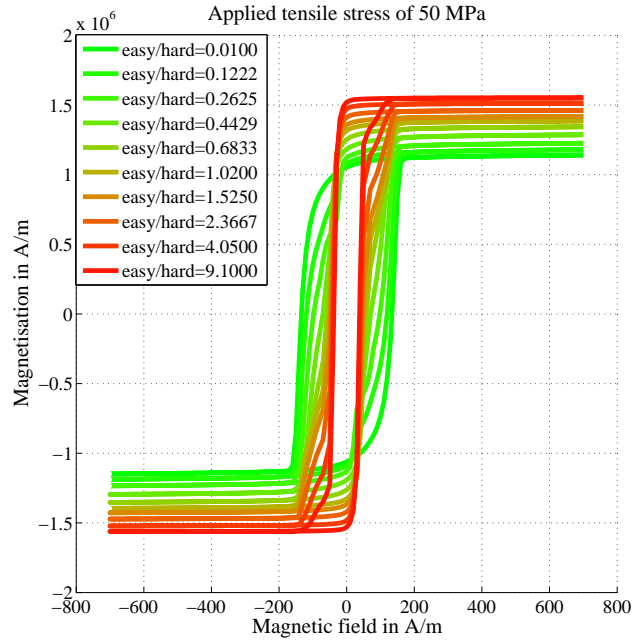

Figure 9: Simulated major loops for $\mathrm{FeSi} 3 \%$ with a tension of $50 \mathrm{MPa}$ for different crystallographic magnetic textures by changing the weight of the grain with an easy axis aligned with the applied field and the weight of the grain with a hard axis aligned with the applied field.

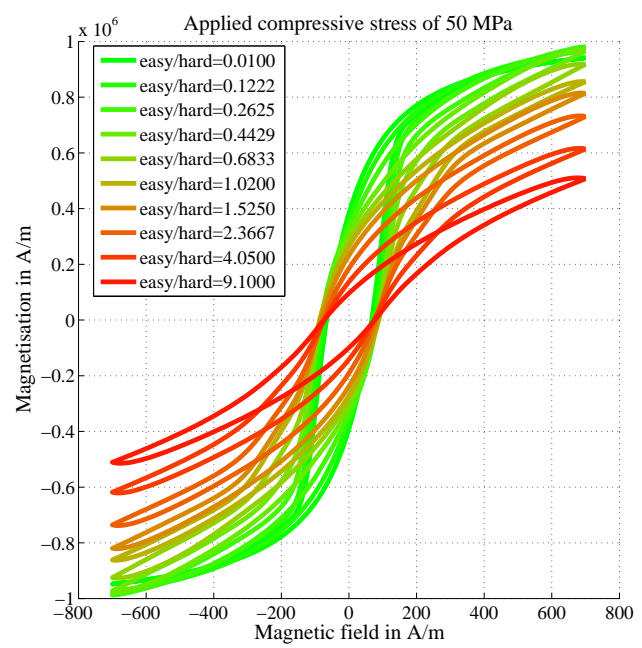

Figure 10: Simulated major loops for FeSi3\% with a pressure of $50 \mathrm{MPa}$ for different crystallographic magnetic textures by changing the weight of the grain with an easy axis aligned with the applied field and the weight of the grain with a hard aligned with the applied field.

pressure of $50 \mathrm{MPa}$, respectively. The different crystallographic magnetic textures are simulated using different weight distributions, as described in Section 3. The influence of different orientation distribution functions is shown by visualizing the ratio of the volume fraction of grains with an easy axis aligned with the externally applied field direction and the volume fraction of grains with a hard axis aligned with the external applied field direction (easy/hard in Fig. 9 and Fig. 10).

The volume fraction of grains with a medium axis aligned with the externally applied field direction remains constant (mean of the volume fraction of grains with an easy axis and the volume fraction of grains with a hard axis aligned with the externally applied field direction). The volume fraction of 


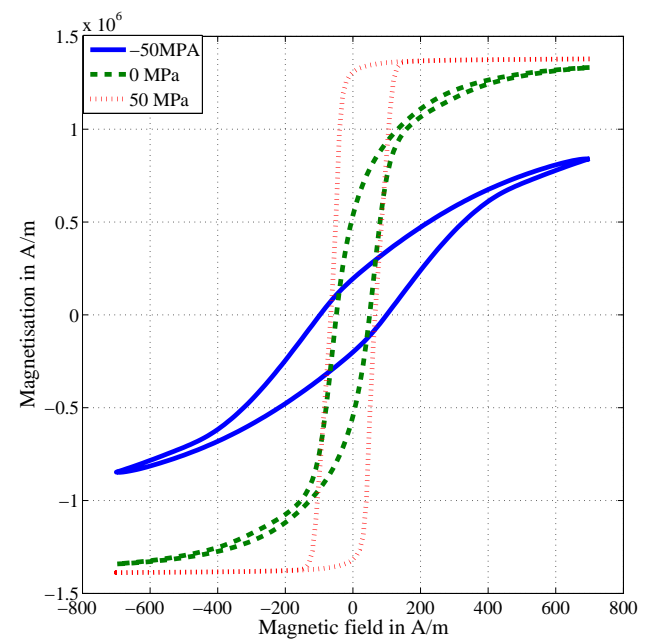

Figure 11: Simulated major loops for FeSi3\% with different mechanical stresses of $-50,0$ and $50 \mathrm{MPa}$, using a measured crystallographic magnetic texture. A positive mechanical stress represents a tensile stress, whereas a negative mechanical stress represents a compressive stress.

grains with a direction which does not correspond to an easy, a medium or a hard direction are obtained using an interpolation between these two directions.

The behaviour of coercivity and remanent magnetisation in Fig. 9 and Fig. 10, corresponding to the crystallographic magnetic texture and mechanical stress, depicts two distinct tendencies. In case of tension (Fig. 9), the coercivity and the remanent magnetisation change both in function of the crystallographic magnetic texture. The remanent magnetisation increases when the easy axis becomes the more pronounced direction, whereas the coercivity decreases corresponding to more square-shaped hysteresis loops. In case of pressure (Fig. 10), only the remanent magnetisation changes in function of the crystallographic magnetic texture, while the coercivity remains constant.

\section{Conclusion}

In this paper, an energy-based multi-scale material model has been extended by inserting a hysteresis energy density function at the grain scale. This model allows to simulate the magnetic behaviour in case of a 3D magnetisation and a multiaxial mechanical stress without the need of tedious parameter identification work as it is the case when a phenomenological model is constructed based on macroscopic measurements data. The material model is based on different material levels (polycrystalline, grain, crystal) defined to simulate the magnetomechanical interactions. The poly-crystalline scale is a representative volume element of the modelled material and exhibits effective material properties. The grain scale consists of multiple crystals, which are almost perfectly aligned with each other, allowing to assume homogeneous elastic properties. The domain scale consists of perfectly aligned magnetic moments. This allows to assume an uniform magnetisation and allows to calculate the Zeeman, the magnetocrystalline anisotropy and the mechanical stress induced anisotropy energy density functions, together with the newly introduced hysteresis energy density function combined with a separate Boltzmann distribution.

The used hysteresis energy density function in the separate Boltzmann distribution increases the obtained probability of the anhysteretic part in favour of the direction closest to the grain scale magnetisation in the previous time step. This, together with the self-consistent scheme, results in the hysteresis loop of the poly-crystalline magnetisation and magnetostriction for different poly-crystalline materials.

The validation of the material model shows a qualitative agreement with the measurements found in literature. As shown in Sec. 5, the energy-based multi-scale material model enables to predict the effect of different mechanical states, be it tension or pressure, on the magnetic behaviour of the material. The change in permeability as well as the deformation of the hysteresis follow the predictions of Bozorth [7, Chapter 13] and Bulte [9] on positive magnetostrictive materials. The model predicts the square shaped hysteresis loop when applying a high tension as well as the diamond shaped hysteresis loop when applying a high pressure.

Using the same mechanism, the effect of the mechanical stress on the magnetostriction loops can be explained. Only when magnetic domain moments have to rotate (switch form easy axes), contribute to the magnetostriction and their maximum contribution is obtained when they have to rotate over an angle of $90^{\circ}$ which requires the higher field.

The basic mechanisms for the hysteresis behaviour, as described in Section 4.2.2, are used to obtain the magnetic and magnetostrictive hysteresis loop as well as their minor loops.

The effect of the crystallographic magnetic texture on the hysteresis loop, Ie., distribution of the different grains, plays an important role in the residual mechanical stress which depends on the applied magnetic field. This gives rise to different magnetisation curves of the same material for the same applied mechanical stress.

This energy based multi-scale material model enables to make qualitatively predictions for: (i) the magnetic properties, (ii) the elastic properties and (iii) the magnetostrictive properties of a material which is subjected to a multi-axial mechanical stress and the inclusion of the hysteresis effect. Making this a promising tool for optimizing the material used in highly efficient but light weighted designs of electromechanical energy transducers.

\section{ACKNOWLEDGEMENTS}

The work of D. Vanoost is funded by the Agency for Innovation by Science and Technology in Flanders (IWT), by a Travel Grant for a long stay abroad of the Research Foundation - Flanders (FWO).

The work of S. Steentjes is supported by the DFG and carried out in the research project Improved modelling and characterization of ferromagnetic materials and their losses. 


\section{REFERENCES}

[1] C. Appino, G. Durin, V. Basso, C. Beatrice, M. Pasquale, and G. Bertotti Effect of stress anisotropy on hysteresis and Barkhausen noise in amorphous materials. Journal of Applied Physics, 85(8):4412-4414, 1999.

[2] W. D. Armstrong. The magnetization and magnetostriction of Tb0.3Dy07Fe1.9 fiber actuated epoxy matrix composites. Materials Science and Engineering: $B, 47(1): 47-53,1997$.

[3] W. D. Armstrong. A directional magnetization potential based model of magnetoelastic hysteresis. Journal of Applied Physics, 91(4):2202-2210, 2002

[4] W. D. Armstrong. An incremental theory of magneto-elastic hysteresis in pseudo-cubic ferro-magnetostrictive alloys. Journal of Magnetism and Magnetic Materials, 263(12):208 - 218, 2003.

[5] M. Bali, H. De Gersem, and A. Muetze. Finite-element modeling of magnetic material degradation due to punching. Magnetics, IEEE Transactions on, 50(2):745-748, 2014.

[6] L. Bernard, X. Mininger, L. Daniel, G. Krebs, F. Bouillault, and M. Gabsi. Effect of stress on switched reluctance motors: A magneto-elastic finiteelement approach based on multiscale constitutive laws. Magnetics, IEEE Transactions on, 47(9):2171-2178, 2011.

[7] R. M. Bozorth. Ferromagnetism (The Bell Telephone Laboratories. The Bell Telephone Laboratories series). Van Nostrand, 6 edition, 1968.

[8] O. E. Buckley and L. W. McKeehan. Effect of Tension Upon Magnetization and Magnetic Hysteresis in Permalloy. Phys. Rev. A., 26:261-273, Aug 1925.

[9] D. Bulte and R. Langman. Origins of the magnetomechanical effect. Journal of Magnetism and Magnetic Materials, 251(2):229 - 243, 2002.

[10] J. M. D. Coey. Magnetism and Magnetic Materials. 2010. Cambridge Books Online.

[11] R. Corcolle, L. Daniel, and F. Bouillault. Generic formalism for homogenization of coupled behavior: Application to magnetoelectroelastic behavior. Phys. Rev. B, 78:214110, 2008

[12] B. D. Cullity and C. D. Graham. Introduction to Magnetic Materials. Wiley-IEEE Press, 2 edition, 2008.

[13] M. Dabrowski and T. Zgodzinski. Some effects of unidirectional stress in si-fe sheets. Physica Scripta, 40(4):514, 1989.

[14] L. Daniel, O. Hubert, and R. Billardon. Homogenisation of magnetoelastic behaviour: from the grain to the macro scale. Computational $\mathcal{E}$ Applied Mathematics, 23(2-3):285-308, 2004.

[15] L. Daniel, O. Hubert, N. Buiron, and R. Billardon. Reversible magnetoelastic behavior: A multiscale approach. Journal of the Mechanics and Physics of Solids, 56(3):1018 - 1042, 2008.

[16] L. Daniel, M. Rekik, and O. Hubert. A multiscale model for magnetoelastic behaviour including hysteresis effects. Archive of Applied Mechanics, 84(9-11):1307-1323, 2014.

[17] A. DeSimone and R. D. James. A constrained theory of magnetoelasticity. Journal of the Mechanics and Physics of Solids, 50(2):283 - 320, 2002

[18] A. DeSimone, R. V. Kohn, S. Müller, F. Otto, et al. Magnetic microstructures-a paradigm of multiscale problems. 1999.

[19] J. D. Eshelby. The determination of the elastic field of an ellipsoidal inclusion and related problems. Proceedings of the Royal Society of London A: Mathematical, Physical and Engineering Sciences, 241(1226):376-396, 1957.

[20] A. Furuya, J. Fujisaki, Y. Uehara, K. Shimizu, H. Oshima, and T. Matsuo. Micromagnetic hysteresis model dealing with magnetization flip motion for grain-oriented silicon steel. Magnetics, IEEE Transactions on, 50(11):1-4, Nov 2014

[21] H. Hauser. Energetic model of ferromagnetic hysteresis: Isotropic magnetization. Journal of Applied Physics, 96(5), 2004.

[22] P. Helnwein. Some remarks on the compressed matrix representation of symmetric second-order and fourth-order tensors. Computer Methods in Applied Mechanics and Engineering, 190(2223):2753 - 2770, 2001.

[23] R. Hill. Continuum micro-mechanics of elastoplastic polycrystals. Journal of the Mechanics and Physics of Solids, 13(2):89-101, 1965.

[24] L. Hirsinger, G. Barbier, C. Gourdin, and R. Billardon. Application of the internal variable formalism to the modelling of magnetoelasticity. Mechanics of Electromagnetic Materials and Structures, 19:54, 2000.

[25] J. Huber, N. Fleck, C. Landis, and R. McMeeking. A constitutive mode for ferroelectric polycrystals. Journal of the Mechanics and Physics of Solids, 47(8):1663-1697, 1999.
26] O. Hubert, L. Daniel, and R. Billardon. Experimental analysis of the magnetoelastic anisotropy of a non-oriented silicon iron alloy. Journal of Magnetism and Magnetic Materials, 254255(0):352 - 354, 2003.

[27] O. Hubert and S. Lazreg. Multidomain modeling of the influence of plastic deformation on the magnetic behavior. Magnetics, IEEE Transactions on, 48(4):1277-1280, April 2012.

[28] M. LoBue, V. Basso, F. Fiorillo, and G. Bertotti. Effect of tensile and compressive stress on dynamic loop shapes and power losses of FeSi electrical steels. Journal of Magnetism and Magnetic Materials, 196197(0):372 _ 374, 1999.

[29] M. LoBue, C. Sasso, V. Basso, F. Fiorillo, and G. Bertotti. Power losses and magnetization process in FeSi non-oriented steels under tensile and compressive stress. Journal of Magnetism and Magnetic Materials, 215216:124 - 126, 2000

[30] A. Moses. Effects of applied stress on the magnetic properties of high permeability silicon-iron. Magnetics, IEEE Transactions on, 15(6):15751579, Nov 1979.

[31] T. Mura. Micromechanics of Defects in Solids. Comparative Studies in Overseas History. Springer Netherlands, 1987.

[32] L. Néel. Les lois de l'aimantation et de la subdivision en domaines élémentaires d'un monocristal de fer (in french). J. Phys. Radium, 5(12):265-276, 1944.

[33] R. Ogden and D. Steigmann. Mechanics and Electrodynamics of Magneto- and Electro-elastic Materials. CISM International Centre for Mechanical Sciences. Springer, 2011.

[34] O. Perevertov and R. Schäfer. Influence of applied tensile stress on the hysteresis curve and magnetic domain structure of grain-oriented fe-3\% si steel. Journal of Physics D: Applied Physics, 47(18):185001, 2014

[35] V. Permiakov, L. Dupré, A. Pulnikov, and J. Melkebeek. Loss separation and parameters for hysteresis modelling under compressive and tensile stresses. Journal of Magnetism and Magnetic Materials, 272276(0):E553 - E554, 2004. Proceedings of the International Conference on Magnetism (ICM 2003).

[36] M. Rekik. Measurement and modeling of magneto-mechanical dissipative behavior of high yield stress ferromagnetic materials under multiaxial loading: application for high speed generator. Doctoral theses, École normale supérieure de Cachan - ENS Cachan, June 2014.

[37] M. J. Sablik and D. C. Jiles. Coupled magnetoelastic theory of magnetic and magnetostrictive hysteresis. Magnetics, IEEE Transactions on, 29(4):2113-2123, 1993

[38] A. Saito, H. Nakamura, and K. Narita. Effects of tensile stresses on the magnetization processes of non-oriented 3\% silicon steel sheets. Journal of Magnetism and Magnetic Materials, 19(1):69 - 71, 1980.

[39] S. Steentjes, K. Hameyer, M. Bednarz, S. Vogt, W. Volk, J. Dierdorf, and G. Hirt. Influence of material processing steps annealing and cutting on magnetic materials' properties relevant for electrical machine design. In Forming Technology Forum 2013, Herrsching, Bayern, Germany, September 2013. Prof.W.Volk.

[40] E. C. Stoner and E. Wohlfarth. A mechanism of magnetic hysteresis in heterogeneous alloys. Philosophical Transactions of the Royal Society of London. Series A, Mathematical and Physical Sciences, 240(826):599_ 642, 1948.

[41] S. Suwas and R. Ray. Crystallographic Texture of Materials. Engineering Materials and Processes. Springer London, 2014.

[42] J. Thoelke and D. Jiles. Model calculation for determining local energy minima in the orientation of magnetic domains in terbium-dysprosiumiron single crystals. Journal of Magnetism and Magnetic Materials, 104107, Part 2(0):1453 - 1454, 1992. Proceedings of the International Conference on Magnetism, Part $\{$ II $\}$.

[43] E. Villari. Change of magnetization by tension and by electric current. Ann. Phys. Chem, 126:87-122, 1865.

[44] S. E. Zirka, Y. I. Moroz, R. G. Harrison, and K. Chwastek. On physical aspects of the Jiles-atherton hysteresis models. Journal of Applied Physics, 112(4), 2012. 